Aim of the study: The aim of this prospective study was to determine the prevalence of malnutrition and to evaluate a more sensitive marker to assess the nutritional status in patients undergoing RT for head and neck cancer.

Material and methods: The prospective study included 51 (mean age of $57.6 \pm 11.2$ years) patients undergoing RT for head and neck cancer. Malnutrition was defined as weight loss $>5 \%$ of baseline. Results: Forty-six (90.2\%) of 51 patients were male. Malnutrition developed in $33(64.7 \%)$ patients during RT. Mean prealbumin level was significantly lower in patients with malnutrition than in those without malnutrition $(17 \pm 5 \mathrm{~g} / \mathrm{dl}$ vs. $22 \pm 5 \mathrm{~g} / \mathrm{dl}$, respectively, $p=0.004)$. On the other hand, there was no significant difference between the two groups in terms of other nutrition parameters including total protein, albumin, total cholesterol, triglyceride, and glucose $(p>0.05)$. The percentage of weight loss negatively correlated with prealbumin $(r=-0.430, p=0.002)$, but not with other nutrition parameters including total protein, albumin, triglyceride, total cholesterol, HDL cholesterol, LDL cholesterol, and glucose $(p>0.05)$.

Conclusions: The prevalence of malnutrition was high in patients with head and neck cancer. Prealbumin was a more sensitive marker than albumin to assess the nutritional status in these patients.

Key words: albumin, head and neck cancer, malnutrition, prealbumin, radiotherapy.

\section{Prealbumin is a more sensitive marker than albumin to assess the nutritional status in patients undergoing radiotherapy for head and neck cancer}

Dilek Unal, Okan Orhan, Celalettin Eroglu, Bunyamin Kaplan

Department of Radiation Oncology, Erciyes University Medical Faculty, Kayseri, Turkey

\section{Introduction}

Malnutrition and cachexia, which is the final form of malnutrition if it is not treated, are common problems in patients with cancer. The most important result of malnutrition is increased complication and death risk during chemotherapy, radiotherapy (RT), or surgical treatment [1]. Patients with head and neck cancer are among those cancer patients in whom malnutrition is the most frequent. Causes of malnutrition in these patients include the following: (a) tumor or RT-induced catabolic factors such as tumor necrosis factor $\alpha$ (TNF- $\alpha$ ) and interleukins, (b) the reduction in dietary intake caused by factors such as tumor, RT, or chemotherapy-induced dysphagia, mucositis or nausea, and (c) poor eating habits associated with excessive alcohol consumption [2, 3].

Measurement of serum proteins can provide indirect information about the levels of visceral protein. Albumin and prealbumin are among such proteins. Prealbumin is a good marker of visceral protein status and is affected earlier by acute variations in protein balance $[4,5]$. Serum albumin is commonly used as surrogate marker of nutrition; however, its half-life of 21 days makes it only minimally valuable. In conditions in which malnutrition develops in a short time, albumin is not a clinically relevant nutritional marker [4, 5].

The aim of this prospective study was to determine the prevalence of malnutrition and to evaluate a more sensitive marker to assess the nutritional status in patients undergoing RT for head and neck cancer.

\section{Material and methods}

This prospective study was performed in the Department of Radiation Oncology in Erciyes University Medical School. Fifty-one patients with nonmetastatic head and neck cancer were enrolled consecutively in the study. Patients were excluded if they were $<18$ years old, had severe disease such as heart failure and hepatic failure, had a history of any other cancer, or if they refused to give consent. The study protocol was approved by the local ethics committee. Informed consent was obtained from all patients subjected to study procedures.

Malnutrition was defined as weight loss $>5 \%$ of baseline. The subjective global assessment of nutritional status (SGA) is used to assess the nutritional status of patients [6]. Body mass index (BMI) was defined as weight in kilograms divided by height in square meters.

\section{Blood samples}

Blood samples were taken from all patients for laboratory examinations such as complete blood count, serum total protein, albumin, glucose, and total lipid profile before beginning the RT course and after the end of RT. On the 
other hand, blood samples which were taken from patients for prealbumin were immediately centrifuged and stored at $-80^{\circ} \mathrm{C}$. Prealbumin was analyzed by the nephelometric method (Dade Behring, USA).

\section{Radiotherapy and chemotherapy}

Patients were irradiated by using $6 \mathrm{MV}$ Linear Accelerator Beams (Varian CDX 2300). RT was curative for the majority of the patients; curative RT in 32 (62.8\%) patients and adjuvant RT in 19 (37.2\%) patients. Radiotherapy was given through two parallel opposite lateral fields to the cervical lymph nodes as well as primary tumor sites and/or through the anterior field to the inferior cervical and the supraclavicular lymph nodes. It was given in 1.8-2.0 Gy/day doses five days a week by conventional fractionation (total 60-70 Gy by spinal cord protection at $46 \mathrm{~Gy}$ ). Cisplatin $50 \mathrm{mg} /$ week was concomitantly given.

\section{Toxicity evaluation}

Radiotherapy-related acute toxicity was evaluated once a week by National Cancer Institute Common Toxicity Criteria (NCl-CTC) version 2.0 [7]. Severe RT-related toxicities including nausea, radiodermatitis, mucositis, and dysphagia were defined as grade 3 or grade 4 RT-related toxicity.

\section{Statistical analysis}

SPSS 15.0 software was used for the statistical analysis. Continuous variables with normal distribution were presented as mean \pm SD. The median value was used where normal distribution was absent. Qualitative variables were given as percent. Statistical analysis for the parametric variables was performed using the Student's t-test between two groups. The Mann-Whitney $U$ test was used to compare nonparametric variables between two groups. The $\chi^{2}$ test and Fisher exact test were used to compare qualitative data between two groups. The correlation analysis was evaluated by Pearson's correlation test for parametric variables and by Spearman's correlation test for nonparametric variables. A $p$ value of 0.05 was considered statistically significant.

\section{Results}

Mean age of 51 patients was $57.6 \pm 11.2$ years; 46 (90.2\%) of the 51 patients were male. The stage of cancer was stage I in 4 (7.8\%), stage II in 5 (9.8\%), stage III in 13 (25.5\%), and stage IV in 29 (56.9\%) patients. The pathology of cancer was squamous cell carcinoma in 46 (90.2\%) and non-squamous cell carcinoma in 5 (9.8\%) patients. Thirty-two (62.7\%) patients received concomitant chemotherapy while 1 (2.0\%) patient dropped out during chemotherapy. On the other hand, the remaining 18 (35.3\%) patients were not given concomitant chemotherapy. Forty-four (86.3\%) patients had a history of smoking whereas 13 (25.5\%) had a history of alcohol use. The localization of cancer was larynx in 27 (52.9\%), nasopharynx in $11(21.6 \%)$, parotid gland in $5(9.8 \%)$, hypopharynx in 2 (3.9\%), lip in 2 (3.9\%), oral cavity in 1 (2.0\%), maxillary sinus in $1(2.0 \%)$, skin cancer + neck metastasis in $1(2.0 \%)$, and unknown primary cancer in 1 (2.0\%) patient.

Malnutrition developed in 33 (64.7\%) patients during RT. Table 1 shows the comparison of demographic and clinical findings in patients with and patients without malnutrition. ECOG score was significantly more impaired in patients with malnutrition than in those without malnutrition after the end of RT while there was no significant difference between the two groups for it before the beginning of RT. Use of concomitant chemotherapy was more frequent in patients with malnutrition than in those without malnutrition. Although the difference between the two groups was not statistically significant, the RT dose was higher in patients with mal-

Table 1. Comparison of demographic and clinical findings in patients with and patients without malnutrition

\begin{tabular}{|c|c|c|c|}
\hline Parameter & $\begin{array}{l}\text { Patients without malnutrition } \\
\qquad(n=18)\end{array}$ & $\begin{array}{l}\text { Patients with malnutrition } \\
\qquad(n=33)\end{array}$ & $p$ \\
\hline age (year) & $57.4 \pm 10.0$ & $57.8 \pm 11.9$ & 0.912 \\
\hline $\begin{array}{l}\text { sex } \\
\quad \text { male }(\%) \\
\text { female }(\%)\end{array}$ & $\begin{array}{c}18(100 \%) \\
-\end{array}$ & $\begin{array}{c}28(84.8 \%) \\
5(15.2 \%)\end{array}$ & 0.101 \\
\hline smoking (\%) & 17 (94.4\%) & 27 (81.8\%) & 0.209 \\
\hline drinking (\%) & $4(22.2 \%)$ & $9(27.3 \%)$ & 0.483 \\
\hline family history of cancer (\%) & $2(11.1 \%)$ & $6(18.2 \%)$ & 0.409 \\
\hline ECOG score s $^{*}$ & $\begin{array}{c}0: 8(44.4 \%) \\
1: 9(50.0 \%) \\
2: 1(5.6 \%)\end{array}$ & $\begin{array}{c}0: 14(42.1 \%) \\
1: 18(54.5 \%) \\
2: 1(3.0 \%)\end{array}$ & 0.884 \\
\hline ECOG score s* $^{\star *}$ & $\begin{array}{c}0: 0(0 \%) \\
1: 9(50.0 \%) \\
2: 8(44.4 \%) \\
3: 1(5.6 \%)\end{array}$ & $\begin{array}{c}0: 1(3.0 \%) \\
1: 4(12.1 \%) \\
\text { 2: } 22(66.7 \%) \\
3: 6(18.2 \%)\end{array}$ & 0.024 \\
\hline use of concomitant chemotherapy (\%) & $5(27.8 \%)$ & 27 (81.8\%) & $<0.001$ \\
\hline radiotherapy dose & $6422 \pm 483$ & $6715 \pm 515$ & 0.053 \\
\hline
\end{tabular}

*Before beginning of radiotherapy course,

${ }^{* *}$ After the end of radiotherapy 
Table 2. Comparison of nutrition parameters in patients with and patients without malnutrition

\begin{tabular}{|c|c|c|c|}
\hline Parameter & $\begin{array}{l}\text { Patients without malnutrition } \\
\qquad(n=18)\end{array}$ & $\begin{array}{l}\text { Patients with malnutrition } \\
\qquad(n=33)\end{array}$ & $p$ \\
\hline total protein (g/dl) & $7.31 \pm 0.68$ & $7.00 \pm 0.48$ & 0.063 \\
\hline albumin (g/dl) & $3.92 \pm 0.40$ & $3.71 \pm 0.35$ & 0.063 \\
\hline prealbumin (g/dl) & $22 \pm 5$ & $17 \pm 5$ & 0.004 \\
\hline total cholesterol (mg/dl) & $211 \pm 42$ & $199 \pm 51$ & 0.399 \\
\hline triglyceride (mg/dl) & $118(60-392)$ & $115(49-385)$ & 0.585 \\
\hline glucose (mg/dl) & $95(56-146)$ & $90(54-166)$ & 0.782 \\
\hline hemoglobin (g/dl) & $13.8 \pm 2.3$ & $12.2 \pm 1.9$ & 0.011 \\
\hline SGA score after the end of RT & $\begin{array}{c}\text { A: } 7(38.9 \%) \\
\text { B: } 10(55.6 \%) \\
\text { C: } 1(5.6 \%)\end{array}$ & $\begin{array}{c}\text { A: } 1(3.0 \%) \\
\text { B: } 24(72.7 \%) \\
\text { C: } 8(24.2 \%)\end{array}$ & 0.002 \\
\hline
\end{tabular}

SGA: subjective global assessment, RT: radiotherapy

Table 3. Comparison of RT-related toxicities in patients with and patients without malnutrition

\begin{tabular}{lcc} 
Parameter & $\begin{array}{c}\text { Patients without malnutrition } \\
(n=18)\end{array}$ & $\begin{array}{c}\text { Patients with malnutrition } \\
(n=33)\end{array}$ \\
\hline presence of severe nausea (\%) & $1(5.6)$ & $5(15.2)$ \\
presence of severe radiodermatitis (\%) & $6(33.3)$ & $18(54.5)$ \\
presence of severe mucositis (\%) & $0(0)$ & $6(18.2)$ \\
presence of severe dysphagia (\%) & $4(22.2)$ & $26(78.8)$
\end{tabular}

nutrition than in those without malnutrition. On the other hand, there was no significant difference between the two groups in terms of other demographic and clinical parameters including age, gender, smoking, drinking, and family history of cancer $(p>0.05)$. In addition, although the data are not shown in the table, there was no significant difference between the two groups in terms of cancer localization, stage of cancer, and co-morbid diseases ( $p>0.05)$.

Comparison of nutrition parameters in patients with and patients without malnutrition are summarized in Table 2. Levels of prealbumin and hemoglobin were significantly lower in patients with malnutrition than in those without malnutrition. Subjective global assessment score was significantly worse in patients with malnutrition than in those without malnutrition. On the other hand, there was no significant difference between the two groups in terms of other nutrition parameters including total protein, albumin, total cholesterol, triglyceride, and glucose $(p>0.05)$.

Table 3 shows the comparison of RT-related toxicities in patients with and patients without malnutrition. Severe dysphagia was more frequent in patients with malnutrition than in those without malnutrition. On the other hand, there was no significant difference between the two groups in terms of other RT-related toxicities including severe nausea, severe radiodermatitis, and severe mucositis.

The percentage of weight loss positively correlated with SGA score after RT $(r=0.621, p<0.001)$, ECOG score after RT $(r=0.515, p<0.001)$, RT dose $(r=0.283, p=0.044)$, the development of severe radiodermatitis $(r=0.287, p=0.041)$, the development of severe mucositis $(r=0.281, p=0.045)$, the development of severe dysphagia $(r=0.662, p<0.001)$, and use of concomitant chemotherapy $(r=0.535, p<0.001)$, and negatively correlated with prealbumin $(r=-0.430$, $p=0.002$ ), but not with other nutrition parameters including total protein, albumin, triglyceride, total cholesterol, glucose, and hemoglobin, or with clinical and demographic parameters including age, gender, smoking, drinking, and stage of cancer $(p>0.05)$.

\section{Discussion}

Patients with head and neck cancer are among those cancer patients in whom malnutrition is the most frequent. Several methods have been used to define malnutrition in studies related to malnutrition in cancer patients. Some authors used weight loss and BMI [8], while other authors used SGA and anthropometric measurements [9]. Although there is an optimal method of assessment, the method most commonly used is evaluation of weight loss and BMI and it often correlates with the results of the disease [8]. Cancer patients are the patient group with the highest prevalence of proteincalorie malnutrition in hospitalized patients [10]. Approximately one third of patients with head and neck cancer have severe malnutrition. Also approximately one third of the patients develop moderate malnutrition [11]. One thousand five hundred and forty-five cancer patients were included in the study conducted by Pressoir et al. and malnutrition, which is defined as weight loss of $10 \%$ or more, is found in $30.9 \%$ of the patients. Cancer localization in 179 of the 1545 patients 
was head and neck and the frequency of malnutrition in this subgroup of patients was as high as $45.6 \%$ [8]. In a study performed by Pirlich et al. in Germany, the prevalence of malnutrition was found as $37.6 \%$ in oncologic patients [9]. In a study performed by Bozzetti et al. in Italy, significant weight loss ( $\geq 10 \%$ ) was observed in $39.7 \%$ of 1000 oncologic patients [12]. Even in patients with early-stage head and neck cancer, prevalence of critical weight loss, which is defined as weight loss of more than 5\% during RT, was observed as approximately $25 \%$ [13]. In our study, malnutrition was defined as weight loss $>5 \%$ of baseline during RT and was observed in 33 (64.7\%) patients. On the other hand, weight loss of more than 10\% during RT was observed in 24 (47.1\%) patients. When the SGA, which is another method of nutritional status assessment, is used to define malnutrition, 15.7\% of 51 patients were well nourished, and $84.3 \%$ malnourished (66.7\% SGA-B and 17.6\% SGA-C) in evaluation after the end of RT. These findings related to the frequency of malnutrition in our study were similar to those regarding the prevalence of malnutrition in patients with head and neck cancer mentioned above.

Measurement of serum proteins can provide indirect information about the levels of visceral protein. Albumin and prealbumin are among such proteins. Prealbumin is synthesized in the liver and acts as a transport protein in the body. It has a shorter half-life of 2-3 days and its amount in the body is low. Therefore, measurement of prealbumin is a good marker of visceral protein status and prealbumin is affected earlier by acute variations in protein balance $[4,5]$. Serum albumin is commonly used as a surrogate marker of nutrition; however, its half-life of 21 days makes it only minimally valuable. With such a long half-life, a new steady state level can only be reached after 100 days [4]. As in this study, in conditions in which malnutrition develops in a short time, albumin is not a clinically relevant nutritional marker. Therefore, prealbumin is a more sensitive marker than albumin or transferrin to assess the nutritional status [4, 5]. Similarly, in this study, prealbumin level was significantly lower in patients with malnutrition than in those without malnutrition and there was no significant difference between the two groups in terms of albumin levels. In addition, the percentage of weight loss that was used to define malnutrition negatively correlated with prealbumin, but not with albumin.

The degree of malnutrition is related to the patient's nutritional status before tumor development, to the characteristics of the tumor, and to the cancer treatment itself such as RT and chemotherapy [14]. Malnutrition is a serious problem in patients with head and neck cancer. It relates closely to the degradation of the quality of life and patient performance, decrease in adherence to treatment and response to therapy, reduction in life expectancy, and longer duration of hospitalization. It also may increase the risk of infection, and treatment toxicity and treatment costs $[1,8,15]$. Performance scores in the patients in this study were in agreement with these findings. ECOG score was significantly worse in patients with malnutrition than in those without malnutrition after RT while there was no significant difference between the two groups for it before RT.

Subjective global assessment (as well as the percentage of weight loss and BMI) is a parameter used to assess the nutritional status in both cancer patients and patients without cancer [9]. Therefore, in this study, it was an expected finding that patients with malnutrition had a worse SGA score.

Radiotherapy has serious side effects in both the early and late period on swallowing functions and these side effects manifest as dysphagia. In the early period, side effects including severe dry mouth, stomatitis, superficial mucosal ulceration, taste disorder, bleeding, pain, and mucositis may develop [16]. On the other hand, in the late period, side effects including osteoradionecrosis, trismus, oral flora changes, dental caries, taste changes, and strictures may occur [17]. As expected, toxicity also increases with increasing dose of RT. In our study, dysphagia, a serious toxicity of RT, was significantly more frequent in patients with malnutrition, so it is a contributing factor to malnutrition in patients with head and neck cancer. Similarly, the development of severe mucositis and severe nausea was more common in patients with malnutrition, but the difference was not statistically significant. However, there was a trend towards elevation which might reach significance if the number of patients is increased. On the other hand, there was also found a significant correlation between the amount of weight loss during RT and the development of RT-related toxicity such as mucositis and dysphagia.

Chemotherapeutic drugs may negatively affect nutrition. This is usually a result of the adverse effects on the oral cavity, oropharynx and esophagus mucosa and hence mucositis and odynophagia may develop. Other side effects that contribute to malnutrition and cachexia include nausea and vomiting. Cisplatin is a commonly used agent in patients with head and neck cancer and has a very high potential for nausea. Combined chemoradiotherapy may increase dysphagia and malnutrition more due to the combined toxicities of the two treatment modalities [1]. Thirty-two patients received concomitant chemotherapy in this study. The chemotherapeutic agent used was cisplatin. As expected, 81.8\% (27 patients) of the 33 patients with malnutrition underwent concurrent chemotherapy, or in other words, 27 of the 32 patients who received cisplatin developed malnutrition. These side effects of cisplatin possibly appear to contribute to malnutrition in our patients.

In conclusion, malnutrition was a common complication in patients undergoing radiotherapy for head and neck cancer. Prealbumin is a more sensitive marker than albumin to assess the nutritional status.

\section{Limitations}

The presented studied group is rather small and heterogeneous. In a study performed in a more homogeneous group of patients (e.g. patients with hypopharynx cancer), probably more unequivocal results can be obtained. However, the number of patients will be even lower. This problem can be overcome by future multicenter studies.

The authors declare no conflict of interest.

This study was supported by Erciyes University, Scientific Research Projects Fund (TST-09-664). 


\section{References}

1. Nitenberg G, Raynard B. Nutritional support of the cancer patient: issues and dilemmas. Crit Rev Oncol Hematol 2000; 34: 137-68.

2. Todorov P, Cariuk P, McDevitt T, Coles B, Fearon K, Tisdale M. Characterization of a cancer cachectic factor. Nature 1996; 379: 739-42.

3. Casas-Rodera P, Gómez-Candela C, Benítez S, Mateo R, Armero M, Castillo R, Culebras JM. Immunoenhanced enteral nutrition formulas in head and neck cancer surgery: a prospective, randomized clinical trial. Nutr Hosp 2008; 23: 105-10.

4. Geisler JP, Linnemeier GC, Thomas AJ, Manahan KJ. Nutritional assessment using prealbumin as an objective criterion to determine whom should not undergo primary radical cytoreductive surgery for ovarian cancer. Gynecol Oncol 2007; 106: 128-31.

5. Guerra LT, Rosa AR, Romani RF, Gurski RR, Schirmer CC, Kruel CD. Serum transferrin and serum prealbumin as markers of response to nutritional support in patients with esophageal cancer. Nutr Hosp 2009; 24: 241-2.

6. Detsky AS, McLaughlin JR, Baker JP, Johnston N, Whittaker S, Mendelson RA, Jeejeebhoy KN. What is subjective global assessment of nutritional status? JPEN J Parenter Enteral Nutr 1987; 11: 8-13.

7. Cancer Therapy Evaluation Program Common Toxicity Criteria, Version 2.0, March 23, 1998.

8. Pressoir M, Desné S, Berchery D, et al. Prevalence, risk factors and clinical implications of malnutrition in French Comprehensive Cancer Centres. Br J Cancer 2010; 102: 966-71.

9. Pirlich M, Schütz T, Norman K, et al. The German hospital malnutrition study. Clin Nutr 2006; 25: 563-72.

10. Nixon DW, Heymsfield SB, Cohen AE, Kutner MH, Ansley J, Lawson $\mathrm{DH}$, Rudman D. Protein-calorie undernutrition in hospitalized cancer patients. Am J Med 1980; 68: 683-90.

11. Goodwin WJ Jr, Byers PM. Nutritional management of the head and neck cancer patient. Med Clin North Am 1993; 77: 597-610.

12. Bozzetti F; SCRINIO Working Group. Screening the nutritional status in oncology: a preliminary report on 1,000 outpatients. Support Care Cancer 2009; 17: 279-84.

13. Nourissat A, Bairati I, Samson E, et al. Predictors of weight loss during radiotherapy in patients with stage I or II head and neck cancer. Cancer 2010; 116: 2275-83.

14. Gaziano JE. Evaluation and management of oropharyngeal dysphagia in head and neck cancer. Cancer Control 2002; 9: 400-9.

15. Norman K, Pichard C, Lochs H, Pirlich M. Prognostic impact of disease-related malnutrition. Clin Nutr 2008; 27: 5-15.

16. Arcuri MR, Schneider RL. The physiological effects of radiotherapy on oral tissue. J Prosthodont 1992; 1: 37-41.

17. Cooper JS, Fu K, Marks J, Silverman S. Late effects of radiation therapy in the head and neck region. Int J Radiat Oncol Biol Phys 1995; 31: 1141-64.

\section{Address for correspondence}

\section{Dr. Dilek Unal}

Erciyes Üniversitesi Tip Fakültesi

M. Kemal Dedeman Onkoloji Hastanesi

Talas Yolu Üzeri

38280, Kayseri, Turkey

tel. 90-3522076666

fax 90-3524375807

Submitted: $\quad 3.10 .2012$

Accepted: $\quad 27.11 .2012$ 